\title{
La convivencia pacífica en la escuela como imperativo de la educación actual: propuestas y perspectivas.
}

Peaceful coexistence at school as an imperative of current education: proposals and perspectives.

DOI: 10.32870/sincronia.axxv.n79.33a21

Ana Cecilia Valencia Aguirre

Departamento de Filosofía. Universidad de Guadalajara (MÉXICO)

CE: anaval a@hotmail.com / ID ORCID: 0000-00015766-712X

María Teresa Prieto Quezada

Universidad de Guadalajara (MÉXICO)

CE: materesaprieto@yahoo.com.mx / ID ORCID: 0000-0002-3299-2927

José Claudio Carrillo Navarro

Unidad de Apoyo a Comunidades Indígenas. Universidad de Guadalajara (MÉXICO)

CE: jccn1964@gmail.com / ID ORCID: 0000-0002-5320-7545

Esta obra está bajo una Licencia Creative Commons Atribución-NoComercial 4.0 Internacional

Recibido: $20 / 09 / 2020$

Revisado: 02/10/2020

Aprobado: $10 / 11 / 2020$

\section{RESUMEN}

El presente trabajo tiene como propósito describir las condiciones de la violencia en los contextos escolares y el imperativo de formar docentes capaces de desarrollar ambientes de convivencia pacífica en las escuelas y las aulas. Asimismo, se plantean principios rectores para guiar la acción práctica, reconociendo las condiciones de los educadores y las experiencias de intervención situadas en escuelas de educación básica en la entidad. Se parte de un marco analítico y descriptivo, donde se recuperan tanto diagnósticos como estudios abordados desde la mirada de diversos teóricos en el campo de la violencia escolar, en este sentido, se enfatiza más en sus resultados como aportes al fenómeno. Esto se justifica ante la demanda de subrayar la necesidad de formar en los docentes de escuelas básicas habilidades sociales y de construcción de ambientes para la convivencia pacífica como condición del desarrollo armónico de los sujetos en condición escolar. 
Palabras Clave: Violencia escolar. Convivencia pacífica. Docentes.

\begin{abstract}
The purpose of this paper is to describe the conditions of violence in school contexts and the imperative to train teachers to transform peaceful coexistence environments in schools and classrooms. Likewise, the guiding principles are proposed to guide the practical action, thinking about the conditions of the educators and starting from intervention experiences located in basic education schools in the entity. It starts from an analytical and descriptive framework, where both diagnoses and studies approached from the perspective of various theorists in the field of school violence are recovered. The intervention proposal has been implemented in school situations and its results are more emphasized as contributions to the phenomenon. This is justified by the demand to underline the need to train in the teachers of basic schools' social skills and the construction of environments for peaceful coexistence as a condition of the harmonious development of the learners.
\end{abstract}

Keywords: School violence. Peaceful coexistence. Teachers.

\title{
Introducción
}

Atender el fenómeno de la violencia escolar no es una propuesta sencilla, por eso consideramos que es necesario centrarnos en los formadores, actores clave, ya que ellos viven experiencias cotidianas y directas con los educandos, conocer sus condiciones reales y sus límites acerca del conocimiento y la comprensión de la violencia escolar es una prioridad. Por otro lado, no es posible entender el fenómeno, sin describir sus dimensiones sociales, culturales y escolares, por eso dedicamos un apartado a los diagnósticos tanto nacionales, internacionales como estatales de la situación actual de la violencia escolar, situado el fenómeno en el caso del bulling o violencia entre pares, por ser, de acuerdo a dichos diagnósticos, el fenómeno más recurrente y grave por sus consecuencias negativas en los educandos. 
En siguientes apartados, se plantean principios que se sugieren para guiar las acciones de intervención ante el fenómeno de la violencia escolar, cabe advertir que como principios rectores no son prescripciones sino ejes orientadores de la acción. Asimismo, se abordan analíticamente las percepciones que los docentes de educación básica tienen y que se recuperan de estudios e intervenciones llevadas a cabo en escuelas primarias de la entidad.

Las diversas investigaciones, en el contexto tanto nacional e internacional, hacen énfasis en una formación de docentes para generar ambientes que propicien la cultura de la paz en las escuelas. Sin embargo, como muestran nuestros estudios, también tenemos que recuperar el saber de los docentes, donde se asientan creencias, quizá la más generalizada es que la violencia proviene de las familias y, por tanto, es tarea de los padres atenderla. Esto ha llevado a consecuencias poco afortunadas, quizá la más recurrente es la trivialización del fenómeno en los contextos tanto áulicos como escolares.

Aunque, de acuerdo a Lucio, Prieto y Carrillo (2018), los docentes, en su mayoría, mencionan el interés en atender e intervenir asertivamente la problemática, ellos muestran su desconcierto ante la falta de apoyos, recursos y asesoría. La tarea es grande y en ella deberían intervenir diversos agentes de la sociedad civil, directivos, gestores, comunidad escolar, padres de familia, entre otros. Las conclusiones son reflexivas e invitan a la tarea de pensar y actuar ante el fenómeno, considerado uno de los principales obstáculos para un desarrollo armónico desde las escuelas, espacios por excelencia formadores de la ciudadanía reflexiva y de sujetos para la convivencia pacífica.

\section{Problematización: La geografía de la violencia en las escuelas}

En la sociedad actual, instituciones como la familia y la escuela siguen siendo coparticipes fundamentales en la tarea de la educación de las nuevas generaciones. No obstante, de acuerdo con Epstein (2002) los proyectos de unos y de otros pueden ser diferentes entre sí, en sus fines o en los medios empleados para obtenerlos. Este autor, analiza el papel de ambas en este proceso, pero sobre todo las formas en que los niños y adolescentes van marcando su percepción de la violencia 
ante la generación adulta. En ese tenor, la exposición a modelos violentos, especialmente durante la infancia y juventud, conduce a la justificación de la conducta violenta, e incrementa el riesgo de ejercerla. El maltrato entre pares, también conocido como bullying, en inglés, se traduce en formas diversas de agresión psicológica, verbal o física, las cuales se producen en forma reiterada a lo largo de un tiempo determinado. En el caso de México, se registra un considerable incremento frente a un contexto social donde la violencia se vuelve una realidad cada vez más cotidiana. La regresión a formas a convivencias menos civilizadas señala De Vargas (1999) es evidente ante el triunfo de valores más individualistas.

Ante ello se sugieren estrategias colaborativas y participativas que movilicen a los actores de la escuela, a fin de desarrollar la capacidad de los alumnos de valorarse, de escuchar a los otros, de respetar, de saber negociar y de resolver pacíficamente los conflictos, en el marco de reglas claras aplicables a la comunidad escolar en su totalidad. Este autor (De Vargas, 1999) distingue cuatro tipos de violencia entre los jóvenes:

- La que proviene de las profundidades de lo psíquico, relacionada con el instinto de conservación, la afirmación de sí y la voluntad de poder.

- La que proviene de conductas lúdicas o de juego.

- La que proviene de agentes adultos como los educadores o los familiares como parte de respuestas con fuerza desmedida.

- La que puede ser calificada como cultural, asociada con concepciones más o menos primitivas que entran en conflicto con las que privan en la sociedad.

Ante estas tipologías de la violencia, se considera el fenómeno del bullying o la violencia entre pares como uno de los mayores problemas del país, de acuerdo a las estadísticas recopiladas en 2017, México es el país del mundo que más casos de bullying registra al año. La Organización para la Cooperación y el Desarrollo Económico (OCDE) estima que unos 18,8 millones de alumnos de educación básica han padecido acoso alguna vez. (Organización de las Naciones Unidas para la Educación, la Ciencia y la Cultura UNESCO, 2017, párr. 4). 
Por su parte, la organización Confianza e Impulso Ciudadano indicaron que México sigue siendo el líder en problemas de bullying a nivel mundial, señaló Luis Wertman, presidente de la organización. (El Financiero, s.d.).

Según la Comisión Nacional de Derechos Humanos (CNDH) en México el número de menores afectados aumentó en 10\% en los dos últimos años. Es decir, casi 7 de cada 10 sufren algún tipo de violencia. El $40.24 \%$ de los estudiantes declaró haber sido víctima de acoso. Un $25.35 \%$ haber recibido insultos y amenazas. Mientras que un $17 \%$ golpes y $44.7 \%$ dijo haber tenido experiencias de violencia verbal, psicológica, física, incluso a través de las redes sociales. (Valdez, 2014).

Mientras la violencia física contra niños y niñas sufrida en la familia decrece a medida que aumenta su edad, ésta se incrementa en el contexto escolar. En ese estudio, dos terceras partes de los niños y niñas de escuelas básicas en México dijeron haber recibido al menos una agresión física durante los dos últimos años. Además, la violencia emocional - en ambos contextos, familia y escuela, aumenta con la edad, lo cual se evidencia con el hecho de que los participantes del estudio dijeron de manera espontánea que lo primero que cambiarían de su escuela son las situaciones frecuentes de violencia que viven en ella.

Por su parte, la secretaria de Educación Jalisco (SEJ) informo que le han reportado 22 casos de enero a junio del 2019 de violencia entre iguales en el ámbito escolar, registrados en educación básica. También se destacó en una encuesta realizada a poco más de 39,000 estudiantes, que en el estado el 17 por ciento de los menores en las primarias y el 14 por ciento en secundarias son víctimas de maltrato por parte de sus pares. (Larios, 2019).

Al interpretar estos datos es posible dar cuenta de que la escuela no es un escenario de convivencia donde priva necesariamente la armonía, sino que muchos contextos se pueden convertir en espacios de conflicto y malestar para ciertos alumnos, por una situación inaceptable tanto a nivel escolar como social. Sencillamente la violencia no debería existir. Por otra parte, en materia de protección de los derechos de los niños, niñas y jóvenes en México, la formulación de leyes que regulen el comportamiento de los sujetos que conviven en las instituciones escolares públicas y privadas es aún incipiente. En este sentido, el artículo noveno de la Ley General de 
Educación de Jalisco establece la obligación del Estado de tomar medidas que aseguren al educando la protección y cuidados necesarios para preservar su integridad física psicológica y social sobre la base de respeto a su dignidad. Sin embargo, ante la emergencia de nuevas formas de violencia escolar, se requiere un proyecto de ley dirigido a lograr condiciones para la convivencia sana y la acción y prevención ante cualquier tipo de agresiones escolares.

\section{Principios orientadores de la intervención sobre el maltrato entre alumnos}

Quienes trabajamos en la educación escolar debemos fomentar en los niños, adolescentes y jóvenes, cierto grupo de ideas clave, o valores guía, a través de la reflexión sobre el acontecer cotidiano. El respeto por el entorno y por todos los seres con los que conviven en él, tiene que ser una prioridad en la formación de los alumnos. En este sentido, coincidimos en que la educación debe fomentar tres sentimientos que van formando la personalidad de los alumnos desde temprana edad: la compasión, la indignación ante lo injusto y el respeto a la diferencia. El primero de éstos nos hace reconocer al otro como un prójimo, como un semejante, y reaccionar en su ayuda ante situaciones que demanden nuestra solidaridad, como el dolor físico, psicológico o social. El segundo, la indignación ante lo injusto, procede del valor ético de la justicia, que obliga al reconocimiento de la dignidad de las personas, a la protesta, la oposición y la acción ante circunstancias donde tal dignidad se vea pisoteada. Finalmente, el respeto a la diferencia adquiere su mejor expresión en la tolerancia, que significa dejar ser a las personas y no agredirlas por el hecho de ser diferentes.

Uno de los principales retos de las actuales generaciones de profesores es hacer frente a la normalización de la violencia entre niñas y niños, es decir, a la insensibilidad creciente en ellos hacia la violencia. En contraparte, se necesitan estrategias para detener el maltrato en la escuela, con apego a la cultura de respeto a la dignidad humana. La violencia que viven actualmente en la escuela los niños, adolescentes y jóvenes es una violación a sus derechos fundamentales, por lo que erradicarla es compromiso y responsabilidad de todos. 


\section{Percepción de los profesores ante la violencia}

En el estudio "Violencia y escuela: hacia la comprensión de sus manifestaciones e imaginarios presentes en la relación maestro-estudiante" (Pinzón, Rodríguez, Riveros y Martínez, 2002), que aborda la comprensión que los profesores tienen de la violencia escolar, se encontró que los profesores poseen una serie de concepciones que refieren, en primer lugar, al hecho de que "la violencia proviene de las condiciones externas en que viven los estudiantes" (p.320) como maltrato o falta de afecto y acompañamiento en procesos de formación de niñas y niños. En otras palabras, cuando en el entorno hay violencia, ésta se traslada a la escuela y que, por tanto, es al interior de las familias donde se debe tratar el problema y no en la escuela.

Una cuestión que emerge de lo anterior es la de la definición de las funciones del docente, en términos de dilucidar si le corresponde atender a los conflictos que se presentan en los distintos espacios escolares. En el estudio citado, se arrojan como conclusiones, que:

a) Los docentes no están preparados para enfrentar situaciones de violencia, como las que supone enfrentarse con alumnos demasiado agresivos $o$ con problemas de comportamientos disruptivos.

b) Los docentes y la institución escolar centran toda su labor y su estructura en la enseñanza de contenidos programáticos, algo que sin embargo para los alumnos no tiene sentido, por lo que finalmente se deben establecer maneras de control cada vez más fuertes sobre ellos, lo que termina convirtiendo a la escuela más en un centro de control que de aprendizaje y enseñanza.

Algunos de los problemas o conflictos que los profesores del nivel básico enfrentan en la cotidianidad, no se han constituido aún en objeto de investigación. Las situaciones que enfrentan a diario los docentes son resueltas a partir de sus propias intuiciones, tradiciones escolares y supuestos, más no parten de un proceso sistemático de reflexión y conocimiento, a partir de lo cual se pueden favorecer estrategias para su atención por parte de los docentes. Ellos, no investigan 
regularmente la naturaleza de los procesos ni las causas de los problemas que enfrentan. No existe una prioridad que posibilite la necesidad de estudiar los aspectos que se presentan en su práctica.

Existe una multiplicidad de situaciones de naturaleza social en la actividad escolar que los docentes no consideran que constituya parte de sus prioridades de trabajo o de intervención. En gran medida y producto de la formación de estereotipos, los roles asumidos obedecen más al señalamiento institucional, que a lo que las problemáticas cotidianas convocan y que, si bien lo afectan, ellos, los docentes, no siempre están preparados para atender.

El determinar si una problemática les corresponde a ciertos sujetos atender o no, en el caso de los docentes, corresponde en ocasiones a una cierta división del trabajo y de los objetos de reflexión que la estructura social y económica impone a la comunidad escolar. En ese sentido las cosas que los docentes tienen que atender y pensar se encuentran delimitadas por los mecanismos de distribución social del poder y el control. Los docentes son actores fundamentales en la transformación favorable del escenario, ya que como señala Tenti (2009):

Los docentes deberán ser cada vez más 'expertos en cultura de las nuevas generaciones' en la medida de que la transmisión de la cultura escolar (el currículum) deberá tener en cuenta no solo las etapas biopsicológicas del desarrollo... sino también las diversas culturas y relaciones con la cultura que caracteriza a los destinatarios de la acción pedagógica (p. 52).

A pesar de que los problemas de violencia escolar, forman parte de un escenario poco alentador, en el caso de Jalisco, el panorama es también grave, ya que según la Secretaria de Educación Jalisco (2019), nueve de cada 10 niños y niñas sufre violencia, este fenómeno alcanza a la población escolar, en el contexto de la educación básica, en las grandes ciudades, pues proliferan una serie de conductas y situaciones de violencia que hasta hace poco eran poco tenidas en cuenta en los estudios y acciones institucionales como aspectos característicos de la población estudiantil, por un lado, y como focos problemáticos sobre los cuales volcar la atención y emprender programas de mejora. (Larios, 2019). 
La falta de referencia a estos problemas, a nivel institucional, y su correlato con la falta de programas de formación de profesores en la línea de la gestión de conflictos y del aprendizaje de actitudes que favorezcan el desarrollo de la conciencia en los actores del proceso educativo en los términos que establecen instancias mundiales como la UNESCO, designados por conceptos como educación para la paz y educación para la convivencia, aspectos que se encuentran favorecidos por las políticas educativas en diversos países como Brasil, Francia y España, representa un desfase más en el caso de nuestro país con respecto a las tendencias internacionales, donde llega el apoyo a este a cuentagotas y no tanto porque fuera obligatorio incorporarse a ellas, sino porque debería representar una orientación prioritaria para comprender y buscar alternativas de solución a un problema que en nuestras escuelas se vuelve cada vez más lacerante.

Dejar fuera del inventario de prioridades de atención, en el terreno educativo, esta situación, por las razones que se haga, implica una fuerte contradicción con el propio discurso oficial en el aspecto de la calidad educativa, en tanto niega las condiciones fundamentales para generar ambientes de aprendizaje propicios para alcanzarla.

Un nuevo problema, significa y provoca la necesidad de pensarlo de manera distinta. Las herramientas con los que no logramos comprender una nueva situación implican la necesidad de realizar nuevas propuestas para escudriñarlo, para realizar intervenciones alternativas, no para instaurar elegantes maquinarias de la inmovilización.

Sensibilizar a los docentes sobre la necesidad de invertir reflexión e intervención de estrategias paradigmáticamente no consideradas en su hacer, que abonen al asunto de una formación para la convivencia dirigida hacia sí mismos y hacia sus estudiantes tendría que ser una de las prioridades fundamentales de la escuela de hoy.

En la actualidad pareciera que la prioridad más importante en la formación de los profesores reside en que cuenten con procesos de actualización disciplinar y/o pedagógico acorde a los requerimientos del desarrollo de la ciencia y la técnica, y que además se constituyan en usuarios de los recientes avances de las tecnologías del aprendizaje. La preeminencia de estos aspectos de formación para los profesores, en detrimento de la que deberían poseer en aspectos de orden 
social, emocional y de convivencia, puede constituirse una de las explicaciones al hecho de que prevalezca una especie de indiferencia en los propios profesores hacia el fenómeno de la violencia, la que ocurre en la "aldea global", o en el entorno cotidiano, pero sobre todo la que tiene lugar en el espacio concreto de la escuela.

¿Cuáles son los saberes de los docentes sobre esta problemática? ¿Qué papel juegan los fines educativos en esta perspectiva? ¿Los docentes dentro del proceso de enseñanza y aprendizaje no están inmersos y a su vez afectados en problemáticas sociales y de convivencia? ¿Hasta dónde la escuela tiene responsabilidad en favorecer o generar el desarrollo de habilidades para enfrentar situaciones de violencia? ¿Qué explica que el profesor no tome conciencia, o no la muestre, en torno a su rol dentro de la problemática de la formación de los estudiantes en habilidades sociales, o más específicamente, qué determinantes influyen para que los profesores tomen o no como parte de su rol la educación para la convivencia? ¿Cómo generar una toma de conciencia en los profesores acerca de la existencia del conflicto y las formas de violencia en los espacios escolares? ¿Qué alcances reales puede tener un programa de formación de profesores en la atención a la problemática de la violencia escolar? ¿Qué estrategias podrían favorecer el desarrollo de habilidades en los docentes para mejorar las relaciones de convivencia y cooperación en los alumnos dentro del aula?

Estas interrogantes, no se plantean para ser respondidas, sino para dimensionar algunas aristas de la complejidad del problema, partimos de que el profesor es un agente que favorece, a través de los distintos procesos de socialización propios de la actividad escolar, la conservación y desarrollo de las estructuras y relaciones que orientan la vida social en un contexto determinado. De ellas se desprenden algunas prioridades que consideramos deberían tomarse en cuenta ante cualquier propuesta de intervención:

1. La identificación de los supuestos, creencias presaberes, o teorías implícitas, que los docentes tienen acerca de las situaciones de conflicto que interfieren en los procesos de convivencia escolar. 
2. La detección, desde la perspectiva de los docentes, de algunas de las necesidades de formación para atender problemáticas sociales que afecten dichos procesos de convivencia.

3. Capacitación, orientación, seguimiento y guía, a través de tutores, en la detección oportuna y el diseño de propuestas de intervención dirigida a la formación de profesores para atender las situaciones de conflicto y violencia que ocurren en el espacio escolar.

\section{Educar para la no violencia. De la teoría a la práctica}

La intervención sobre un problema tan extendido y complejo como el del maltrato entre alumnos, además de requerir la participación de diversos actores de la comunidad escolar, implica contar con una serie de ideas y de prácticas clave para prevenir o atender el problema. Algunas de estas ideas y prácticas, que sugerimos promover en las interacciones profesor-alumno y alumno-alumno propias del trabajo cotidiano escolar son, entre otras:

- El rechazo a las imágenes violentas, así como a las demostraciones innecesarias de fuerza.

- El fomento de la cultura de respeto a la diferencia y de solidaridad con las personas y grupos vulnerables.

- La exploración y atención de casos de maltrato entre iguales en la comunidad escolar.

- El reconocimiento y el uso de la autoridad institucional para implementar normas de convivencia.

- La necesidad del trabajo de integración de grupos y la enseñanza de competencias sociales.

En lo relacionado con la intervención, sea como prevención o como forma de solución de conflictos y atención a las situaciones de maltrato entre alumnos, se pueden emplear diversas técnicas y programas, de acuerdo con los niveles educativos y con los contextos sociales donde se desarrolla la labor escolar. Al respecto, Cohen, 2003; Ortega y Del Rey, 2003; Viñas, 2007; Coronado 2008, dan distintas aportaciones teóricas a este tema, así como nuestra experiencia en el estudio y la intervención sobre el maltrato entre alumnos nos sugieren que es necesaria una formación específicamente orientada hacia la comprensión de este problema por parte de profesores y 
alumnos. En otras palabras, no es posible resolver conflictos, educar para la paz y la convivencia, si no se reconoce, en principio, la existencia del problema, su naturaleza y dimensiones.

En un trabajo anterior (Lucio, Prieto y Carrillo, 2018), encontramos que, por un lado, los alumnos vinculados a esta situación, independientemente del rol que jueguen en ella (víctimas, agresores o espectadores) reconocen los efectos del maltrato sobre quienes lo sufren, pero también, preocupantemente, que muchos de ellos, al tomar el rol de agresores, experimentan satisfacción. Esto nos pone en alerta ante el posible hecho de que las formas violentas de convivencia, donde se incluye el maltrato entre alumnos, sean percibidas como algo natural, lo cual debería ser un motivo de reflexión y de rechazo.

La estrategia se basa una serie de acciones tendientes a provocar en profesores y alumnos una reflexión de fondo sobre lo que representa el problema, por qué es un problema y qué medios hay para hacerle frente, desde una perspectiva que se alimente de las propias respuestas de los actores. Se trata de sensibilizarlos básicamente sobre la experiencia de quienes son víctimas, sobre los valores en juego en casos particulares de maltrato entre alumnos, y dar una panorámica de las acciones que a nivel individual, institucional y social se pueden llevar a cabo para que el problema disminuya.

En este sentido, sugerimos, con base en nuestros trabajos de intervención desarrollados en escuelas básicas (Prieto, Carrillo y Jiménez, 2009) dos metodologías de intervención. Una, el taller de formación para la mediación y negociación y la otra, basada en el uso didáctico de la narrativa, donde se recuperan resultados de investigación de campo (entrevistas y cuestionarios realizados a niños de primaria) víctimas de maltrato por sus pares debidas a razones como sus diferencias religiosas, étnicas, físicas y económicas. Los resultados originales del trabajo de campo se convertirán luego en historias, mediante la dinámica El árbol de la vida (terapia narrativa) donde se planteen formas de trabajo en los grupos escolares y realizando una propuesta de formación para docentes.

En nuestras intervenciones, la narrativa cobra un singular sentido, como lo ha señalado Valencia: 
La narrativa es un recurso importante no sólo para investigar la subjetividad de los actores educativos, además es un elemento para la catarsis de quienes han vivido experiencias de violencia; o bien mostrar los motivos de los victimarios; la revelación de situaciones vividas se convierte en elemento reflexivo que conduce a analizar la propia vida y a asumir un juicio moral ante la vivencia experimentada y reflexionada desde el sujeto que se narra, que se configura una voz y un rostro ante el otro, que se hace visible ante el mundo de su propio relato o bien se inventa una máscara propia del mundo social; que asume el recorrido como trayecto de diferencia desde la comprensión con los otros (Valencia, 2015, p. 7)

Así, la propuesta de intervención más adecuada, de acuerdo a nuestras experiencias en el campo, son aquellas donde los propios sujetos, desde sus vivencias propias, resignifican y asumen juicios reflexivos para cambiar su entorno desde una nueva actitud. Este proceso no es simple, requiere acompañamiento, asesoría y apoyo.

\section{Algunas de las percepciones del docente sobre la violencia escolar}

En nuestros trabajos de intervención realizados en contextos de escuelas de educación básica (Prieto y Valencia, 2015 y Prieto, Carrillo y Jiménez, 2009) inferimos que:

- Pocos docentes le dedican momentos significativos a tratar la cuestión de la violencia dentro de las aulas y no existen elementos para concluir si se trata de una estrategia preventiva o correctiva.

- En general, los docentes utilizan el dialogo para enfrentar estas situaciones, pero su palabra es diferente dentro del aula que fuera de ella, al interior desarrollan una función correctiva, mientras que fuera del aula, un tono más dialógico. La mayoría señala emplearlo en la clase, si bien falta por detallar el contenido y forma de los mensajes, y el impacto del contexto social del aula durante este tipo de intervención, a diferencia de la que ocurre cuando el profesor habla con el alumno fuera de la clase. 
- En general los docentes tienen buena predisposición para tratar estos temas de la violencia en las aulas, pero refieren que hace falta la intervención y el compromiso de las familias.

- La mayoría de los docentes señala el acoso entre alumnos como la problemática principal de violencia en las aulas.

- Respecto a la postura de los profesores acerca del bullying, como un problema fundamental dentro de la convivencia en la escuela y a las circunstancias asociadas con su ocurrencia, el problema es percibido por los profesores, aunque no da cuenta del modo en que ven que su participación incide sobre él, promoviendo o inhibiendo determinadas normas y prácticas de socialización en las cuales puede existir una postura especifica hacia esta forma de agresión.

- Otro de los aspectos claves que generan violencia tiene que ver con la intolerancia dentro del ámbito escolar que involucra a niños, y niñas por igual. Resultado de la ausencia de un valor importante en la vida social actual, y como consecuencia, proyecta de manera significativa la posibilidad de una educación para la convivencia, cuyos agentes centrales fuesen los profesores. Sin embargo, esta posibilidad choca ante obstáculos de carácter institucional, como la falta de estímulos a quienes participaran en un programa de este tipo.

- Un aspecto por destacar, sin duda, tiene que ver con la reiterada referencia que los docentes hacen a la agresión de la que son víctimas.

Por lo anterior, se puede decir que todos los maestros son conscientes de su papel para generar habilidades de convivencia, sin embargo, la mayoría manifiesta que los programas y contenidos de estudio se centran en generar habilidades de conocimiento, pero no de relaciones con el otro; "al educando lo hemos atiborrado de conocimientos y hemos descuidado las habilidades y las aptitudes" (Prieto, Carrillo y Jiménez, 2009, p. 54). Es decir, existe la percepción, pero no se desarrollan métodos para implementar, por lo menos una o dos ocasiones a lo largo del semestre, una clase centrada en la importancia de habilidades para la no violencia y prácticas de convivencia. Todos los docentes, sin excepción, admitieron su compromiso y la responsabilidad de promover en sus alumnos este tipo de aptitudes, pero demandan capacitación, apoyo y acompañamiento. 
Algunas de las competencias y habilidades que, consideramos, deben desarrollar los profesores, y que a su vez ellos consideraron fundamentales para desarrollar su labor, son las siguientes:

1. Desarrollo personal: por medio del cual se exterioriza la motivación para progresar en el espacio escolar, con actitudes y comportamientos asertivos y la aceptación de desafíos que revelan autoestima, autoconfianza y adaptación, factores todos de la inteligencia emocional.

2. Trabajo en equipo: la habilidad para trabajar con otros y lograr los objetivos, hacer y cumplir los planes y tomar las decisiones adecuadas de manera conjunta, que es también parte de la competencia emocional.

3. Promover solución de problemas, individualmente o en equipo: la comprensión que se tenga de los mismos, la elaboración de un plan y su ejecución. Esta visión integral es lo que exige el mundo productivo a los profesionales de cualquier área para reconocer sus competencias.

4. Educar en la paz y no violencia, basándonos en los derechos humanos, en la democracia y formar en valores, tolerancia y respeto a la diferencia.

5. Mejorar la convivencia en el contexto escolar, educando en la prevención, previsión, y protección de las niñas y niños buscando alternativas de solución no violentas.

6. Prevenir la violencia y lograr la resolución pacífica de conflictos mediante el autoconocimiento, el respeto al prójimo y al próximo.

7. Establecer límites y correctivos saludables ante situaciones iniciales de violencia entre pares, en tiempo y forma.

Es necesario, por otra parte, señalar que estas habilidades son imperativos, resultado de análisis de los fenómenos de la violencia expresados y referidos por los propios actores en las escuelas como acciones necesarias para construir una convivencia pacífica que reestructure el tejido escolar e incida en el entorno social. 


\section{A manera de conclusión}

A lo largo de este trabajo, hemos transitado del diagnóstico a la propuesta. Y en esta última, a mostrar los imperativos, las demandas y desafíos de construir un entorno para la convivencia pacífica desde los propios sujetos de la comunidad escolar.

Podemos considerar que la escuela, la familia, los docentes, el gobierno, el sector privado y la sociedad civil deben ofrecer a niños y niñas, recursos, tiempo y espacios dignos y de calidad para que se desarrollen actividades, además de que se fomenten actitudes y valores basados en la responsabilidad y el respeto por el otro, promoviendo una cultura de no violencia y de solución pacífica de los conflictos en un entorno educativo en el que el maltrato e intimidación entre pares y sus efectos destructores desaparezcan. Se trata de romper un esquema donde la fuerza mayor destruye a las personas, y sustituirlo por uno donde se aceptan diferencias y normas para garantizar el respeto por los otros.

El reto es aceptar el desafío de formar educadores para la paz, que rescaten su saber docente y permitan, desde el mismo, resignificarlo. Por ello es desde las experiencias situadas en escuelas y comunidades escolares donde se podría desarrollar el análisis y la reflexión de los problemas de violencia que tanto alumnos como docentes han vivido y viven cotidianamente.

La perspectiva que surge de un modelo desde los propios sujetos es la más propicia, al respecto, se mencionan en este trabajo dos intervenciones situadas: Un taller de formación para la mediación y negociación; y, un uso didáctico de la narrativa. Ambas propuestas forman un solo entramado, en la voz y la actitud del actor está el problema y es en comunidad como se puede visibilizar y atender desde los propios sujetos. Como podrá verse, la propuesta se sitúa en aprendizajes colaborativos. La posibilidad de transformación está en el reconocimiento de la problemática hecha por los propios sujetos, pero esto, como bien lo han explicitado, demanda espacios, apoyos, intervenciones, acompañamientos de expertos, $\mathrm{y}$, sobre todo, colaboración de familias, gestores, autoridades educativas y civiles. La tarea es compleja, pero confiamos en la posibilidad de lograr avances significativos como ha quedado ya evidenciado en los aportes de diversos estudios e intervenciones. 


\section{Referencias}

Coronado, M. (2008). Competencias sociales y convivencia. Buenos Aires: Novedades Educativas.

Cohen, J. (2003). La inteligencia emocional en el aula: Proyectos, estrategias e ideas. Buenos Aires: Troquel.

De Vargas, P. (1999). Directeur du college de l'Elysée. Francia. Consultado el 12 de febrero de 2020, en http://tafel.levillage.org/societe/violence.html\#chron

Viñas, J. (2007). Conflictos en los centros educativos. Cultura Organizacional y mediación para la convivencia. Barcelona: Editorial Grao.

El Financiero (s.d.). Organización presenta guía contra el acoso escolar. [Archivo de video]. El Financiero. Televisión. https://www.elfinanciero.com.mx/tv/bitacora-politica/organizacionpresenta-guia-contra-el-acoso-escolar

Epstein, J. (2002). Construction des reperes chez l' enfant et prevention des violences et incivilities, Conferencia para la FOCEF, Québec, febrero.

Larios, R. (23 de julio de 2019). SEJ reporta 22 casos de bulllying en 2019. El Universal. Un1ón, Jalisco. Educación. https://www.unionjalisco.mx/articulo/2019/07/23/educacion/sejreporta-22-casos-de-bulllying-en-2019

Ortega R. y Del Rey, R. (2003) El proyecto Bullying de la escuela de Sevilla. Un modelo Educativo. Conferencia Europea.

Organización de las Naciones Unidas para la Educación, la Ciencia y la Cultura UNESCO (2017). Según los datos ofrecidos por PISA 2015 el acoso escolar es una de las mayores preocupaciones en las escuelas. https://es.unesco.org/news/segun-datos-ofrecidos-pisa2015-acoso-escolar-es-mayores-preocupaciones-escuelas

Pinzón, J.; Rodríguez, L.; Riveros, O. y Martínez, S. (2002). "Violencia y escuela: hacia la comprensión de sus manifestaciones e imaginarios presentes en la relación maestro-estudiante", en Barbosa, B. (comp.) El oficio de investigar. Educación y pedagogía frente a nuevos retos, Bogotá: Universidad Pedagógica Nacional, 315-351.

Lucio, L. A.; Prieto, M. T.; \& Carrillo, J. C. (2018). Manifestaciones de violencias entre alumnos de educación superior: Los usos del WhatsApp. Alteridad, 13(2), 204-213. 
Consultado el 10 de octubre de 2019 en https://doi.org/10.17163/alt.v13n2.2018.04

Prieto, M. T.; Carrillo, J.C. y Jiménez, J. (2009). Formación de profesores para la solución de conflictos. Buenos Aires. Novedades Educativas.

Prieto, M. T. y Valencia A. C. (2015). Miradas analíticas sobre temas de subjetividad y violencia en el campo educativo. Perspectivas y propuestas. México: Universidad de Guadalajara. CUCEA. Guadalajara.

Tenti, E., y Tedesco, J. C. (2009). Nuevos discentes: las características sociales y culturales de los destinatarios de la acción educativa. Buenos Aires, Arrgentina.: Departamento de Educación Cooperativa, Fundación Bica. Disponible en: www.pedagogiadelapaz.com.ar

Valdez, B. (23 de mayo de 2014). México es el primer lugar de bullying a escala internacional. Milenio. Opinión. https://www.milenio.com/politica/mexico-es-el-primer-lugar-de-bullyinga-escala-internacional

Valencia, A. C. y Nava, J. M. (2015) La narrativa, elemento intersubjetivo y vía del juicio reflexionante en la acción educativa. México: COMIE. 